\title{
A Design Space for Visualization Onboarding in Data-Driven Stories
}

\author{
Morteza Asgari* \\ Polytechnique Montreal
}

\author{
Thomas Hurtut ${ }^{\dagger}$ \\ Polytechnique Montreal
}

\section{INTRODUCTION}

Data-Driven Stories (DDS) are stories that combine text and data portrayed as visualization in a narrative format $[5,6]$. They are among the popular ways of communicating information by online medias nowadays [3-5]. For DDS authors and designers, it's important to minimize the risk of misinterpreting visualizations by their readers. Visualization onboarding, embedding knowledge and guidance have been meant to provide adequate support for readers to understand visualizations as they progress through DDS [2,7]. Onboarding is a continuous mechanism which involves various DDS elements and interactions on each step. Several previous studies attempted to identify and classify storytelling techniques in DDS [6,8]. While these techniques prospect a satisfactory communication, it's not clear how they can be applied to facilitate the visualization understanding throughout the story. They rather conceptualized different aspects of storytelling individually, and as such, the chronology of onboarding steps has been missed. Although their techniques and design spaces represent a tangible level of abstraction, they will not benefit authors in the story design process. Authors either rely on their guess work or mimic previous DDS to accommodate support in their DDS scenarios. In this project, our overall goal is to propose a multidimensional design space for onboarding techniques in DDS that can benefit to authors during their design process.

\section{Preliminary Methods}

Current definitions of onboarding and related terms all come from the visual analytic field $[1,7]$. We thus first propose a new definition for onboarding in the context of DDS, as In our context, onboarding covers "all the techniques applied by authors that support readers to understand the curated visualizations of narrative storytelling".

We then present a new multidimensional design space to classify Visualization Onboarding Techniques in DDS. In contrast to previous design spaces proposed for DDS [6,8], our method provides a holistic view on onboarding approaches which also later reveals the procedure and sequence of actions occurring in DDS support process. The proposed design space gives authors and designers a practical road map to systematically choose right method of supporting readers in DDS. This design space may create a range of possibilities with various interconnections between visual elements, reader's actions and their intended analysis. It is decomposed along the following main dimensions:

1. Specifications: Contains all semantically related properties to onboarding including level of support and directness of onboarding;

2. Technical: Covers all the aspects that reveal the technicality of onboarding process within a visualization like analytical annotations and visualization cues.

*e-mail: morteza.asgari@polymtl.ca

†e-mail: thomas.hurtut@polymtl.ca
3. Flow: Enumerate different techniques of onboarding that keep the flow of the onboarding process glued between visualizations as well as requirements to resolve and connect continuous visualizations;

4. Behavioral: Refers to all readers' side involvement, behaviors and interactions while onboarding process is ongoing.

\section{Preliminary Findings}

We examined the comprehensiveness of our new design space with a preliminary set of 40 different DDS. Those DDS were selected from popular online journals (e.g. Washington Post, NYT, Telegraph). The correspondence between the dimensions of our design space and these use cases verify the breadth of the onboarding techniques embedded in our design space. This survey also demonstrates the popularity of most used techniques.

In many DDS, we also observed a concurrent emergence of a specific onboarding technique with a visualization chart, functionality or analysis. In addition, our survey reveals the nature of onboarding as a multi-step process which encourages designers to break their tasks into multiple stages rather than cluttering their DDS visualizations with annotations and improperly embedded interactions.

\section{Questions for ATtendeEs}

Besides the feedback on our design space dimensions, we would like to discuss with attendees about evaluation strategies. In order to evaluate our work, our first idea is to conduct case studies among a group of data journalists and designers from a newsroom we collaborate with. Our goal is to portray usability and effectiveness of our design space in a practical context. Qualitative versus quantitative approach, potential relevant metrics etc. are aspects that we also like to have feedback on.

\section{REFERENCES}

[1] D. Ceneda, T. Gschwandtner, T. May, S. Miksch, H.-J. Schulz, M. Streit, and C. Tominski. Characterizing guidance in visual analytics. IEEE Transactions on Visualization and Computer Graphics, 23(1):111-120, 2016.

[2] D. Ceneda, T. Gschwandtner, and S. Miksch. A review of guidance approaches in visual data analysis: A multifocal perspective. In Computer Graphics Forum, vol. 38, pp. 861-879. Wiley Online Library, 2019.

[3] L. T. M. Comet. Visual narrative data-driven storytelling. ACM Transactions on Multimedia Computing, Communications and Applications, 1(1):1-20, 2018.

[4] A. Ojo and B. Heravi. Patterns in award winning data storytelling: Story types, enabling tools and competences. Digital journalism, 6(6):693$718,2018$.

[5] N. H. Riche, C. Hurter, N. Diakopoulos, and S. Carpendale. Data-driven storytelling. CRC Press, 2018.

[6] E. Segel and J. Heer. Narrative visualization: Telling stories with data. IEEE transactions on visualization and computer graphics, 16(6):1139$1148,2010$.

[7] C. Stoiber, F. Grassinger, M. Pohl, H. Stitz, M. Streit, and W. Aigner. isualization onboarding: Learning how to read and use visualizations. In Proc. VisComm, 2019.

[8] C. D. Stolper, B. Lee, N. Henry Riche, and J. Stasko. Emerging and recurring data-driven storytelling techniques: Analysis of a curated collection of recent stories. Technical report, April 2016. 